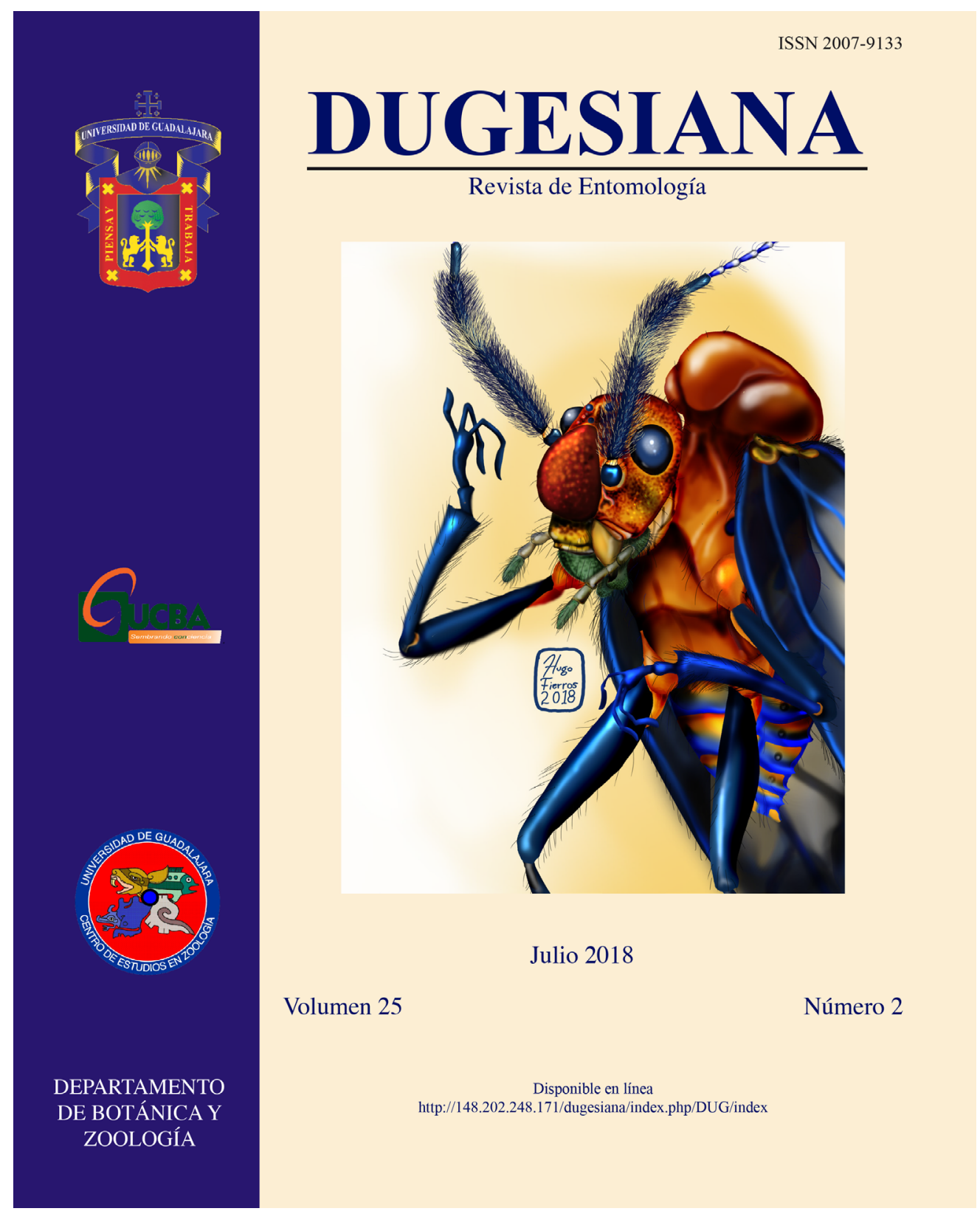

Dugesiana, Año 25, No. 2, julio 2018-diciembre 2018 (segundo semestre de 2018), es una publicación semestral, editada por la Universidad de Guadalajara, a través del Centro de Estudios en Zoología, por el Centro Universitario de Ciencias Biológicas y Agropecuarias. Camino Ramón Padilla Sánchez \# 2100, Nextipac, Zapopan, Jalisco, Tel. 37771150 ext. 33218, http://148.202.248.171/dugesiana/index.php/DUG/index, glenusmx@gmail.com. Editor responsable: José Luis Navarrete Heredia. Reserva de Derechos al Uso Exclusivo 04-2009-062310115100203, ISSN: 2007-9133, otorgados por el Instituto Nacional del Derecho de Autor. Responsable de la última actualización de este número: José Luis Navarrete Heredia, Editor y Ana Laura González-Hernández, Asistente Editorial. Fecha de la última modificación 25 de julio 2018, con un tiraje de un ejemplar.

Las opiniones expresadas por los autores no necesariamente reflejan la postura del editor de la publicación.

Queda estrictamente prohibida la reproducción total o parcial de los contenidos e imágenes de la publicación sin previa autorización de la Universidad de Guadalajara. 


\title{
Aorphallus cibriani gen. nov., sp. nov., y otros Photinini de México (Coleoptera: Lampyridae)
}

\section{Aorphallus cibriani gen. nov., sp. nov., and other Photinini of Mexico (Coleoptera: Lampyridae)}

\section{Santiago Zaragoza-Caballero ${ }^{1}$ e Ishwari Giovanni Gutiérrez-Carranza}

Departamento de Zoología, Instituto de Biología, Universidad Nacional Autónoma de México, Apartado Postal 70-153, 04510 CdMx, México. ${ }^{1 E}$-mail: zaragoza@ib.unam.mx

\section{RESUMEN}

Basado en la forma del edeago, se describen un género y cuatro especies de la tribu Photinini de México: Aorphallus gen. nov., Aorphallus cibriani, sp. nov. de Tenango de Doria, Hidalgo, Ankonophallus consueloae sp. nov. de Ocozococuautla, Chiapas, Photinus (Paraphotinus) acremophallus de Chetumal, Quintana Roo, El Remate, Campeche, Ocosingo, Chiapas y Tuxtepec, Veracruz y $P .(P)$ aulophallus sp. nov. de Zacapoaxtla, Puebla. Aorphallus cibriani sp. nov., se compara con Ankonophallus zuninoi y Pyropyga chemsaki, especies de la tribu Photinini.

Palabras clave. Aorphallus gen. nov., especies nuevas, Photinini.

\begin{abstract}
Acording with the aedeagus morphology, a new genus and four new species are described, Aorphallus cibriani gen. nov., sp. nov., Ankonophallus consueloae sp. nov., Photinus (Paraphotinus) acremophallus and P.(P) aulophallus sp. nov. These taxa belonging to the tribe Photinini, and they are from Tenango de Doria, Hidalgo; Ocozococuautla, Chiapas; Chetumal, Quintana Roo; El Remate Campeche; Ococingo, Chiapas; Tuxtepec, Veracruz y Zacapoaxtla, Puebla. Aorphallus cibriani sp. nov. is compared with Ankonophallus zuninoi and Pyropyga chemsaki, species within Photinini as well.
\end{abstract}

Key words. Aorphallus gen. nov., new species, Photinini.

Zaragoza (1995) caracterizó el aparato reproductor masculino de diversos géneros de la familia Lampyridae como: Magnoculus McDermot, 1964: 36, Phaenolis Gorham, 1880: 10, Tenaspis LeConte, 1881: 33, Cratomorphus Motschulsky, 1853: 35, Aspisoma Laporte, 1833: 145, Aspisomoides Zaragoza, 1997: 34, Photinus Laporte, 1833: 141, Pyropyga Motschulsky, 1853: 28, Lucidota Laporte, 1833: 136, Photuris Dejean, 1833: 163, Bicellonycha Motschulsky, 1853: 54 y Pterotus LeConte, 1859: 86. Posteriormente, Zaragoza (2000b) y ZaragozaCaballero y Navarrete-Heredia (2014), crearon los géneros Pyropygodes y Ankonophallus y los caracterizaron con base en dicha estructura. Estos estudios han mostrado patrones estructurales del aparato reproductor masculino, que los identifica y segrega en forma clara, lo que concuerda con lo mencionado por Green (1961), sobre la importancia de esta estructura en el reconocimiento de los géneros de la familia Lampyridae.

Además, para las especies norteamericanas del género Photinus, Green (1956) estableció dos divisiones considerando la presencia o ausencia de dos excrecencias ("ventro-basal processes") en el edeago. Zaragoza (1995), haciendo referencia a la primera división de Green, creó el subgénero Paraphotinus para incluir las especies del género Photinus que no presentan las excrecencias referidas en el edeago.

Tomando en cuenta esos antecedentes y el estudio de ejemplares provenientes de diferentes estados de México, en este trabajo se describe un género nuevo de la tribu Photinini, Aorphallus gen. nov. y cuatro nuevas especies, Aorphallus cibriani sp. nov., Ankonophallus consueloae sp. nov., Photinus. (Paraphotinus) acremophallus y $P$. (P.) aulophallus y sp. nov., se desconocen aspectos de la biología de las especies.

\section{MATERIAL Y MÉTODO}

Se examinaron ocho ejemplares de Aorphallus cibriani sp. nov., procedentes de Tenango de Doria, Hidalgo; ocho de Ankonophallus consueloae sp. nov. de Ocozocuautla, Chiapas; cinco dePhotinus (Paraphotinus acremophallus) sp. nov. de Chetumal, Quintana Roo, (2); El Remate Campeche (1); Ocosingo, Chiapas (1) y Tuxtepec, Veracruz (1) y dos de P.(P.) aulophallus sp. nov. de Zacapoaxtla, Puebla. A todos los especímenes se les extrajo el aparato reproductor masculino el que fue montado en triángulos de cartón. Así mismo, se tomaron medidas del largo y ancho total, largo y ancho del pronoto, largo y ancho 
de los élitros, distancia inter ocular, largo y ancho del ojo, primero y tercero antenómeros. Se calculó la relación entre largo y ancho de los élitros y entre largo de élitro y largo del pronoto (Cuadro 2). Las figuras se obtuvieron con la ayuda de un microscopio Zeiss AXIO Zoom. V16. Las medidas se expresan en milímetros. Los ejemplares se encuentran depositados en la Colección Nacional de Insectos, Instituto de Biología, Universidad Nacional Autónoma de México, CdMx, México (CNIN).

Aorphallus gen. nov.

http://zoobank.org/3C3AC9E7-8EC6-4B60-893E834AF6BC3756

Diagnosis. Morfológicamente parecido a los géneros Ankonophallus y Pyropyga. Del primero se puede segregar por tener la frente y el clípeo soldados (Fig. 2) y el edeago en forma de bayoneta, con el lóbulo medio curvado (Figs. 9-11); en Ankonophallus la frente y el clípeo están libremente articulados (Fig. 4) y los lóbulos laterales del edeago son curvados, en tanto el lóbulo medio es recto (Figs. 12-14). Del último se distingue por: tener las antenas más largas y casi filiformes y el edeago más largo y esbelto; en Pyropyga las antenas son cortas y serradas y el edeago es robusto. Además, de los dos géneros es diferente por tener la cabeza parcialmente cubierta por el pronoto, el espacio interocular cóncavo, los tubérculos anteníferos prominentes y el clípeo de forma trapezoidal (Fig. 2). Características de tres especies están indicadas en el Cuadro 1.

Especie tipo Aorphallus cibriani sp. nov.

Descripción. Cuerpo paralelo; cabeza vertical, parcialmente cubierta por el pronoto; ojos enteros, casi esféricos, reducidos; antenas largas, rebasando la mitad del cuerpo, aplanadas, de once antenómeros, el primero más corto que los dos siguientes juntos, del 3-10 aplanados, con lados casi paralelos, el undécimo lanceolado, un poco más largo que el precedente; clípeo trapezoidal, soldado a la frente, sutura fronto-clipeal lateralmente marcada; labro membranoso; mandíbulas falcadas, esbeltas; palpos maxilares con cuatro palpómeros, los labiales de tres; pronoto semicircular, con los ángulos posteriores agudos y el borde posterior ondulado; pronoto casi cuadrangular, borde posterior romo; élitros paralelos; tarsómeros cortos, el cuarto bífido; pigidio redondeado, sin órganos fotógenos; último esternito ojival; edeago largo con los lóbulos laterales libremente articulados a la pieza basal, en forma de bayoneta, lóbulo medio alargado, lateralmente comprimido (Figs. 9-11).

Etimología. La palabra Aorphallus está formada por los vocablos Gr. aor = espada, boyoneta y phallus $=$ pene.

Aorphallus cibriani sp. nov.

Figuras 1-2, 9-11

http://zoobank.org/41035691-60AB-4212-9F8A$446 \mathrm{E} 460061 \mathrm{DF}$
Holotipo macho. Largo 46.2, ancho 16.0. Color negro a excepción de: pronoto y los tres últimos segmentos abdominales amarillos; sin aparato luminoso. Cabeza. Corta, más angosta que el pronoto, espacio inter ocular cóncavo, amplio (0.41) dos veces el ancho del ojo (0.2), brillante, rugosamente punteado, piloso (Fig. 2); tubérculos anteníferos prominentes; antenas con el escapo de 0.22 de largo, segundo y tercer segundo antenómero de 0.10 y 0.31 respectivamente, del cuarto al décimo de longitud semejante (0.30), el undécimo ligeramente más largo que el anterior (0.41); ojos reducidos, más largos que anchos ( $0.30 \mathrm{X} 0.22)$; frente vertical, angosta; clípeo con el borde anterior recto, con pocas sedas; sutura frontoclipeal marcada a los lados (Fig. 2); labro membranoso; mandíbulas falcadas, esbeltas, casi desnudas en la base; palpómero maxilar apical securiforme; el último labial securiforme. Tórax. Pronoto (Fig. 1) más ancho que largo (1.39 X 0.80, integumento brillante, piloso, disco convexo; escutelo con el integumento rugoso; élitros (Fig. 1) un poco más de cuatro veces más largos que anchos (3.82 X 0.80), integumento rugosamente punteado y piloso, la pilosidad decumbente, con sedas largas y cortas; patas cortas, fémures angostos, aplanados, tibias igual, tarsómeros cortos, primer metatarsómero un poco más largo que los dos siguientes juntos (0.22 X 0.19), el cuarto bífido, cubriendo la mitad del quinto, uñas simples. Abdomen. Con ocho esternitos visibles, de longitud semejante, borde posterior del pigidio redondo, borde posterior del séptimo esternito redondo; aparato reproductor esbelto (Figs. 9-11). Hembra similar al macho. Largo total 4.33; ancho total 1.56, espacio inter ocular y ojos más reducidos $(0.36),(0.20 \mathrm{X} 0.15)$, el pronoto con una franja longitudinal de color marrón.

Variación. El color del pronoto en dos ejemplares presenta una franja longitudinal de color marrón, la talla varía de 4.39 a 5.42 de largo y de 1.12 a 1.60 de ancho.

Discusión. Considerando el color, forma y tamaño del cuerpo, de las antenas, y sobre todo la ausencia de aparato luminoso, se relaciona con Pyropyga minuta LeConte, 1852: 333, de la que se segrega por la forma muy diferente del aparato reproductor masculino. Otras características diagnósticas se incluyen en el Cuadro 1.

Etimología. Aorphallus cibriani sp. nov. Especie dedicada con respeto y reconocimiento al destacado Dr. David Cibrián Tovar, especialista en entomología forestal.

Holotipo macho etiquetado: México, Hidalgo, Tenango de Doria, La Viejita, TIV2119, 16/VI/2013, col. N. Gutiérrez. Depositado en la CNIN de la Universidad Nacional Autónoma de México. (COL. TIP. 3219). Paratipos seis machos y una hembra mismos datos que el holotipo. . Depositados en la CNIN (COL. TIP. 3220; COL. TIP. 3221; COL. TIP.3222; COL. TIP. 3223; COL. TIP. 3224; COL. TIP. 3225; COL. TIP. 3226) Depositados en la Colección Nacional de Insectos (CNIN). 
Ankonophallus consueloae sp. nov.

Figuras 3-4, 12-14

http://zoobank.org/C996C783-FF21-45DA-BD0244BDC5526103

Holotipo macho. Largo 48.7, ancho 15.8. Color. Lados del pronoto amarillentos, disco con banda negra más amplia en la parte posterior; escutelo negro; élitros negros, con una banda angosta marginal; ventralmente marrón obscuro; mandíbulas, pro y mesofémures con el borde posterior cremoso, últimos ventritos ambarinos; sin aparato luminoso. Cabeza. Corta, más angosta que el pronoto $(0.82 \times 1.34)$, espacio inter ocular plano, más de dos veces lo ancho del ojo (0.18 X 0.48), brillante, rugosamente piloso (Fig. 4); antenas ligeramente serradas, escapo 0.23 de largo, segundo y tercer segundo antenómero 0.12 y 0.25 respectivamente, del cuarto al décimo de longitud semejante (0.36), el undécimo más largo que el anterior (0.41); ojos reducidos, casi tan largos como anchos (0.23 X 0.18); frente vertical, angosta; clípeo transverso, muy corto, con el borde anterior casi recto, con largas sedas a los lados, sutura fronto-clipeal membranosa, completa (Fig. 4); labro membranoso; mandíbulas falcadas, robustas, con grandes sedas en la base; palpos maxilares de cuatro palpómeros, el terminal fusiforme; los labiales con tres, el último securiforme. Tórax. Pronoto (Fig. 3) semicircular, más ancho que largo (1.34 X 1.08), ángulos posteriores agudos, integumento brillante, piloso, disco convexo; escutelo espatulado, punteado y piloso; élitros (Fig. 3) casi paralelos, casi cinco veces más largos que anchos (3.79 $X$ 0.76), con tres costillas longitudinales, la marginal y sutural más cortas, integumento rugosamente punteado y piloso, la pilosidad decumbente, con sedas largas y cortas; patas cortas, fémures angostos, aplanados, tibias igual, metatarsómero ligeramente más largo que los dos anteriores juntos, el cuarto bífido, largo, casi cubriendo al quinto, uñas simples. Abdomen. Con ocho esternitos visibles, de longitud semejante, borde posterior del pigidio truncado, borde posterior del séptimo esternito cóncavo, el octavo con el borde redondo; aparato reproductor con los lóbulos laterales casi triangulares, cubriendo dos tercios del lóbulo medio, éste con dos proyecciones ventro-laterales (Figs. 12-14).

Discusión. Las dimensiones del cuerpo y el patrón de coloración del pronoto de Ankonophallus consueloae, la acercan a Pyropyga exstincta (Gorham), de la que se distingue por las diferencias en el aparato reproductor masculino (ver Zaragoza, 1993 Figs. 17, 43-45; 2014, Figs. $32,19-21)$.

Etimología. Ankonophallus consueloae sp. nov. Es dedicada a Consuelo Gutiérrez, destacada doctora y madre de mi nieta.

Holotipo macho etiquetado: México, Chiapas, Ocozocuatla, $3 \mathrm{Km}$. W Laguna Bélgica $16^{\circ} 53^{\prime} 47.2^{\prime}$; 9302709.5”W, 956msnm, 5.20/VI/2003. Cols V.H. Toledo, A.M. Corona. Depositado en la CNIN de la Universidad
Nacional Autónoma de México. (COL. TIP. 3227). Siete Paratipos machos mismos datos que el Holotipo. Depositados en la CNIN (COL. TIP. 3228; COL. TIP. 3229; COL. TIP. 3230; COL. TIP. 3231; COL. TIP. 3232; COL. TIP. 3233; COL. TIP. 3234).

\section{Photinus (Paraphotinus) acremophallus sp. nov.} Figuras 5-6, 15-17

http://zoobank.org/FDCC66A9-559E-42D7-91CE30CC91478DF7

Holotipo macho. Largo 61.9, ancho 17.8. Color. Cuerpo marrón a excepción de lados del pronoto amarillentos, disco con banda longitudinal marrón, con esbozos de manchas rojizas a los lados y élitros con una banda marginal y sutural cremosa; aparato luminoso ocupando toda la superficie de los esternitos 5-6. Cabeza. Corta, más angosta que el pronoto (1.3 X 1.58), espacio inter ocular cóncavo, un poco más ancho que el ancho del ojo (0.44 X 0.34), brillante, rugosamente punteado y piloso (Fig. 6); antenas filiformes, escapo 0.27 de largo, segundo y tercer antenómero de 0.15 y 0.22 respectivamente, del cuarto $(0.31)$ al décimo disminuyendo paulatinamente en largo, undécimo de 0.26; ojos grandes, más largos que anchos ( $0.59 \times 0.34)$; frente vertical, angosta; clípeo transverso, muy corto, con el borde anterior casi recto, con largas sedas a los lados, sutura fronto-clipeal membranosa, completa (Fig. 6); labro membranoso; mandíbulas falcadas, robustas, con grandes sedas en la base; palpos maxilares de cuatro palpómeros, el terminal fusiforme; los labiales con tres, el último securiforme. Tórax. Pronoto semicircular, más ancho que largo (1.78 X 1.19), ángulos posteriores casi rectos, integumento brillante, piloso, disco convexo; escutelo espatulado, punteado y piloso; élitros casi paralelos, más de cinco veces tan largos como anchos (5.06 X 0.789), integumento chagrinado, punteado y piloso, la pilosidad de dos tipos una, la larga y erecta, otra corta y decumbente; patas cortas, fémures angostos, aplanados, tibias igual, metatarsómero más largo que los dos anteriores juntos, (25,57 X 18.55) el cuarto bífido, corto, casi cubriendo la mitad al quinto, uñas simples. Abdomen. Con ocho esternitos visibles, 5-6 más largos que los anteriores y el posterior, con dos pequeñas manchas transparentes, borde posterior del pigidio redondeado, borde posterior del séptimo esternito cóncavo, el octavo triangular; aparato reproductor con los lóbulos laterales bifurcados casi desde la base, ramas dorsales más cortas que las ventrales, cubriendo dos tercios del lóbulo medio, éste de forma tubular y apicalmente curvado, orificio medio terminal (Figs.15-17).

Discusión. La apariencia del edeago de Photinus (Paraphotinus) acremophallus sp. nov., lo relaciona con: $P$. (P.) texanus (Green), P. (P.) inmmaculatus (Green), $P$. $(P$.$) cooki (Green), P. (P.) marginellus (LeConte), P$. (P.) tuxtlaensis (Zaragoza), P. (P.) furcatus Zaragoza, $P$. (P.) juarezae Zaragoza y $P$. (P.) anisodrilus Zaragoza, que se distinguen por el mayor o menor grado de bifurcación que presentan los lóbulos laterales y, la carencia de 
excrecencias dorso-basales en el lóbulo medio del reproductor masculino. De $P$. (P) furcatus, se distingue por su talla ( 6.19, 1.78. X 5.66, 2.10) (Figs. 15-17).

Etimología. El nombre propuesto alude a la ramificación de los lóbulos laterales y proviene del Griego acremo $=$ ramas y phallus pene.

Holotipo macho etiquetado: México, Km 146 Chetumal-Pto. Júarez, Qna. Roo, 17/III/72, col. A. Ibarra. Depositado en la CNIN de la Universidad Nacional Autónoma de México. (COL. TIP. 3235). Cuatro paratipos machos etiquetados: Km.146 carr. Chetumal-Cancun, Mex. 16/VIII/82. (1) depositado en la CNIN (COL. TIP. 3236; (1); Tuxtepec, Oax. 11/74 (1) Depositado (COL. TIP. 3237); El Remate, Campeche, México, 26/IV/82, col. A. Ibarra (1) Depositado en la CNIN (COL. TIP. 3238); México, Chiapas, Ocosingo Chajul, Reserva Montes Azules, 10 al 16/VII/87 (1) Depositado en la CNIN (COL. TIP. 3239), Cols. F. Arias, R. Barba, L. Cervantes.

Photinus (Paraphotinus) aulophallus sp. nov. Figuras 7-8, 18-20

http://zoobank.org/EEF3C1A7-8702-40F8-93EE59EAFEF7AE9D

Descripción. Holotipo macho Largo 65.5. Ancho 23.9. Color. Cuerpo negro a excepción de los lados del pronoto y borde interno de los fémures que son amarillento, dos manchas rojizas sobre el disco pronotal que presentan una banda longitudinal negra, aparato luminoso en los esternitos 5-6. Cabeza. Corta, más angosta que el pronoto (1.18 X 1.84), espacio inter ocular cóncavo, un poco más amplio que lo ancho del ojo (0.47 X 0.4), brillante, rugoso y piloso (Fig. 8 ); antenas con el escapo de 0.36 , segundo y tercer segundo antenómero de 0.15 y 0.36 respectivamente, del cuarto al décimo disminuyendo paulatinamente, hasta el undécimo (0.3); ojos más largos que anchos ( $0.54 \times 0.4)$; frente vertical, angosta; clípeo transverso, corto, borde anterior casi convexo, con largas sedas a los lados, sutura frontoclipeal membranosa, completa (Fig. 8); labro membranoso; mandíbulas falcadas, con sedas en la base; palpos maxilares de cuatro palpómeros, el terminal fusiforme; los labiales con tres, el último securiforme. Tórax. Pronoto (Fig. 7) semicircular, más ancho que largo (1.84 X 1.21), margen posterior ondulado, ángulos posteriores un tanto agudos, integumento brillante y piloso, disco convexo; escutelo espatulado, gruesamente punteado, piloso; élitros (Fig. 7) aovados, casi cuatro veces y media más largos que anchos (5.34 X 1.19), integumento rugosamente punteado y piloso, la pilosidad decumbente, de dos tipos una larga y otra muy corta; patas cortas, fémures angostos, aplanados, tibias igual, metatarsómero ligeramente más largo que los dos anteriores juntos, el cuarto bífido, largo, casi cubriendo al quinto, uñas simples. Abdomen. Con ocho esternitos visibles, de longitud semejante, con pequeñas manchas traaparentes hacia los lados, borde posterior del pigidio redondo, borde posterior del séptimo esternito recto, el octavo con el borde redondo; aparato reproductor robusto, con los lóbulos laterales anchos, formando un tubo que cubre al lóbulo medio, éste ancho con el orificio medio terminal (Figs. 18-20).

Discusión. El color del pronoto de P. (P.) aulophallus sp. nov. es similar al de $P$. (P) acremophallus sp. nov. del que se distingue por la forma del cuerpo y sobre todo la estructura del reproductor masculino (Figs. 18-20).

Etimología. El epíteto está formado por los vocablos griegos aulo $=$ tubo y phallus $=\mathrm{a}$ pene.

Holotipo macho etiquetado: México, Puebla, Zacapoaxtla, 11/VI/2009, cols. H. Brailovsky, E. Barrera Depositado en la CNIN de la Universidad Nacional Autónoma de México. (COL. TIP. 3240). Paratipo macho mismos datos que el holotipo Depositado en la CNIN. (COL. TIP. 3241). Comentario adicional. En el año 2000 Zaragoza describió Photinus (s.str.) furcatus del estado de Morelos y la ubicó en dicho género al considerar que los individuos de esa especie presentaban dos reducidas excrecencias dorsobasales en el lóbulo medio del edeago. Al revisar más ejemplares de esta especie, así como ejemplares de la serie tipo, se ha reconocido la ausencia de tales estructuras por lo que se ha concluido que dicha especie debe ser ubicada como Photinus (Paraphotinus) furcatus (Zaragoza). Esta especie se suma a otras 14 con la misma característica (Apéndice 1).

\section{LITERATURA CITADA}

Green, J.W. (1956). Revision of the neartic species of Photinus (Coleoptera: Lampyridae). Proceedings of the California Academy of Sciences, 28(15): 561-613.

Green, J.W. (1961). Revision of the species of Pyropyga (Lampyridae). The Coleopterists'Bulletin 15(3): 65-74.

Zaragoza-Caballero, S. (1995). La familia Lampyridae en la Estación de Biología Tropical

"Los Tuxtlas" Veracruz México. Publicaciones Especiales 14, Instituto de Biología, Universidad Nacional Autónoma de México.

Zaragoza-Caballero, S. (2000a). Cantharoidea (Coleoptera) de México. IV. Nuevos Photinus (Lampyridae) del estado de Morelos. Dugesiana, 7 (1): 1-17.

Zaragoza-Caballero, S. (2000b). Cantharoidea (Coleoptera) de México. VI. Un Nuevo género y una nueva especie de Lampyridae del Estado de Morelos. México. Dugesiana, 7 (1): 19-22.

Zaragoza-Caballero, S. y J.L. Navarrete-Heredia. (2014). Descripción de cuatro especies de Ankonophallus gen. nov. (Coleoptera: Lampyridae: Photinini). Dugesiana, 21(2): 125-130. 
Cuadro 1. Características distintivas de Ankonophallus zuninoi, Zaragoza y Navarrete Pyropyga chemsaki Zaragoza y Aorphallus cibriani sp. nov. (Coleoptera: Photinini).

\begin{tabular}{|l|l|l|l|}
\hline Carácter/especie & Ankonophallus zuninoi & Pyropyga chemsaki & $\begin{array}{l}\text { Aorphallus cibriani gen. } \\
\text { nov., sp. nov. }\end{array}$ \\
\hline Cabeza & $\begin{array}{l}\text { convexa, cubierta totalmente } \\
\text { por el pronoto }\end{array}$ & $\begin{array}{l}\text { convexa, cubierta totalmente } \\
\text { por el pronoto }\end{array}$ & $\begin{array}{l}\text { cóncava, cubierta } \\
\text { parcialmente por el pronoto }\end{array}$ \\
\hline Tubérculos anteníferos & no prominentes & no prominentes & prominentes \\
\hline Sutura fronto-clipeal & membranosa & indistinta & indistinta \\
\hline Clípeo & transverso, corto & bilobulado, largo & trapezoidal, largo \\
\hline Antenas & $\begin{array}{l}\text { largas, rebasando medio } \\
\text { cuerpo }\end{array}$ & $\begin{array}{l}\text { cortas, no alcanzando la } \\
\text { mitad del cuerpo }\end{array}$ & $\begin{array}{l}\text { largas, rebasando medio } \\
\text { cuerpo }\end{array}$ \\
\hline Mandíbulas & medianas & robusta & esbeltas \\
\hline Palpómero maxilar apical & ovoide & fusiforme & securiforme \\
\hline Palpómero apical labial & securiforme & securiforme & securiforme \\
\hline Pronoto & $\begin{array}{l}\text { ojival, ángulos posteriores } \\
\text { casi rectos }\end{array}$ & $\begin{array}{l}\text { semicircular, ángulos } \\
\text { posteriores agudos }\end{array}$ & $\begin{array}{l}\text { semicircular, ángulos } \\
\text { posteriores agudos }\end{array}$ \\
\hline Séptimo esternito & escotado & escotado & truncado \\
\hline Octavo esternito & largo, & corto, ojival & corto, ojival \\
\hline Lóbulo medio & largo, esbelto, recto & corto, robusto & $\begin{array}{l}\text { largo, lateralmente } \\
\text { comprimido, curvado }\end{array}$ \\
\hline Lóbulos laterales & $\begin{array}{l}\text { cortos, curvos, romos, } \\
\text { lejando descubierto al } \\
\text { lóbulo medio }\end{array}$ & $\begin{array}{l}\text { largos, basalmente } \\
\text { lóbendiculados, cubriendo al }\end{array}$ & $\begin{array}{l}\text { largos, triangulares, } \\
\text { cubriendo al lóbulo medio }\end{array}$ \\
\hline
\end{tabular}


Cuadro 2.- Morfometría de cuatro especies de Photinini de México (Holotipo; M. media).

\begin{tabular}{|c|c|c|c|c|}
\hline $\begin{array}{l}\text { Especie/ } \\
\text { carácter }\end{array}$ & $\begin{array}{l}\text { Aorphallus cibriani } \\
\text { gen. nov., sp. nov. }\end{array}$ & $\begin{array}{l}\text { Ankonophallus } \\
\text { consueloae sp. nov. }\end{array}$ & $\begin{array}{l}\text { Paraphotinus } \\
\text { acremophallus sp. } \\
\text { nov. }\end{array}$ & $\begin{array}{l}\text { Paraphotinus } \\
\text { aulophallus sp. nov. }\end{array}$ \\
\hline $\begin{array}{l}\text { Largo total } \\
\text { M }\end{array}$ & $\begin{array}{l}4.62 \\
4.99\end{array}$ & $\begin{array}{l}4.87 \\
4,46\end{array}$ & $\begin{array}{l}7.17 \\
5.90\end{array}$ & $\begin{array}{l}6.55 \\
6.80 \\
\end{array}$ \\
\hline $\begin{array}{l}\text { Ancho total } \\
M\end{array}$ & $\begin{array}{l}1.60 \\
1.46\end{array}$ & $\begin{array}{l}1.58 \\
1.35\end{array}$ & $\begin{array}{l}2.17 \\
1.81\end{array}$ & $\begin{array}{l}2.39 \\
2.47\end{array}$ \\
\hline $\begin{array}{l}\text { Largo pronoto } \\
\text { M }\end{array}$ & 0.80 & $\begin{array}{l}1.08 \\
0.93\end{array}$ & $\begin{array}{l}1.45 \\
1.15\end{array}$ & $\begin{array}{l}1.21 \\
1.26\end{array}$ \\
\hline $\begin{array}{l}\text { Ancho pronoto } \\
\mathrm{M}\end{array}$ & $\begin{array}{l}1.39 \\
1,37\end{array}$ & $\begin{array}{l}1.34 \\
1.19\end{array}$ & $\begin{array}{l}2.00 \\
1.58\end{array}$ & $\begin{array}{l}1.84 \\
1.88\end{array}$ \\
\hline $\begin{array}{l}\text { Largo élitros } \\
\text { M }\end{array}$ & $\begin{array}{l}3.82 \\
4.09\end{array}$ & $\begin{array}{l}3.79 \\
3.53\end{array}$ & $\begin{array}{l}5.73 \\
4.76\end{array}$ & $\begin{array}{l}5.34 \\
5.53\end{array}$ \\
\hline $\begin{array}{l}\text { Ancho élitros } \\
M\end{array}$ & 0.80 & $\begin{array}{l}0.76 \\
0.67\end{array}$ & $\begin{array}{l}1.08 \\
0.90\end{array}$ & $\begin{array}{l}1.19 \\
1.22\end{array}$ \\
\hline $\begin{array}{l}\text { Distancia interocular } \\
\text { M }\end{array}$ & $\begin{array}{l}0.41 \\
0.40\end{array}$ & $\begin{array}{l}0.48 \\
0.43\end{array}$ & $\begin{array}{l}0.53 \\
0.43\end{array}$ & $\begin{array}{l}0.47 \\
0.49\end{array}$ \\
\hline $\begin{array}{l}\text { Largo ojo } \\
M\end{array}$ & 0.30 & $\begin{array}{l}0.23 \\
0.23\end{array}$ & $\begin{array}{l}0.69 \\
0.56\end{array}$ & $\begin{array}{l}0.56 \\
0.52\end{array}$ \\
\hline $\begin{array}{l}\text { Ancho ojo } \\
M\end{array}$ & $\begin{array}{l}0.20 \\
0-18\end{array}$ & $\begin{array}{l}0.18 \\
0.14\end{array}$ & $\begin{array}{l}0.39 \\
0.36\end{array}$ & $\begin{array}{l}0.40 \\
0.36\end{array}$ \\
\hline $\begin{array}{l}\text { Antenómero } 1 \\
M\end{array}$ & $\begin{array}{l}0.22 \\
0.21\end{array}$ & $\begin{array}{l}0.23 \\
0.26\end{array}$ & $\begin{array}{l}0.41 \\
0.33\end{array}$ & $\begin{array}{l}0.36 \\
0.36\end{array}$ \\
\hline $\begin{array}{l}\text { Antenómero 2 } \\
M\end{array}$ & $\begin{array}{l}0.10 \\
0-09\end{array}$ & $\begin{array}{l}0.10 \\
0.09\end{array}$ & $\begin{array}{l}0.14 \\
0.14\end{array}$ & $\begin{array}{l}0.15 \\
0.15\end{array}$ \\
\hline $\begin{array}{l}\text { Antenómero } 3 \\
\text { M }\end{array}$ & $\begin{array}{l}0.31 \\
0.24\end{array}$ & $\begin{array}{l}0.25 \\
0.24\end{array}$ & $\begin{array}{l}0.30 \\
0.24\end{array}$ & $\begin{array}{l}0.36 \\
0.31\end{array}$ \\
\hline $\begin{array}{l}\text { Elitros largo/ancho } \\
\text { M }\end{array}$ & $\begin{array}{l}4.77 \\
5.61\end{array}$ & $\begin{array}{l}4.98 \\
5.24\end{array}$ & $\begin{array}{l}5.30 \\
5.27\end{array}$ & $\begin{array}{l}4.48 \\
4.15\end{array}$ \\
\hline $\begin{array}{l}\text { Elitros largo/ pronoto } \\
\text { largo } \\
\text { M }\end{array}$ & $\begin{array}{l}4.77 \\
5-02\end{array}$ & $\begin{array}{l}3.71 \\
3.08\end{array}$ & $\begin{array}{l}3.95 \\
4.53\end{array}$ & $\begin{array}{l}4.41 \\
4.37\end{array}$ \\
\hline
\end{tabular}



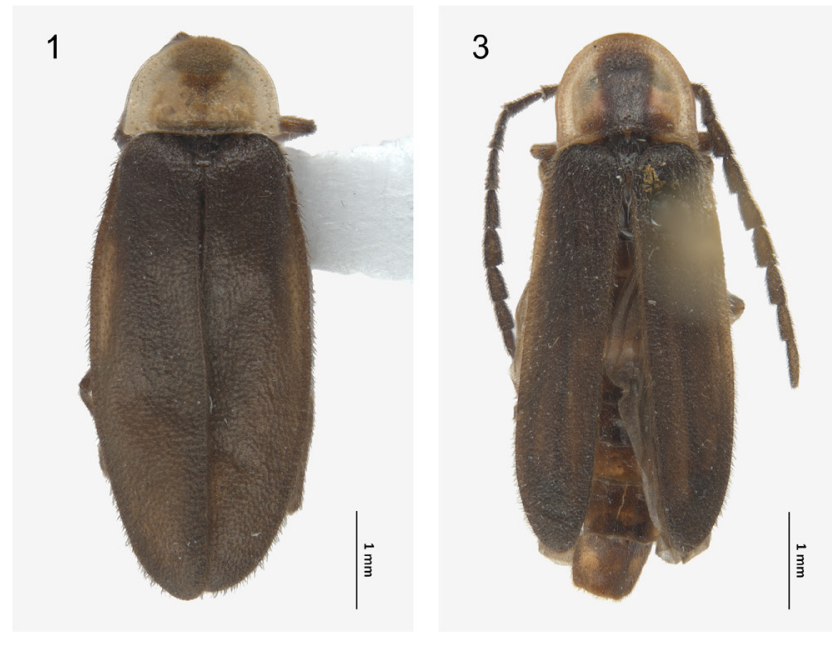

2

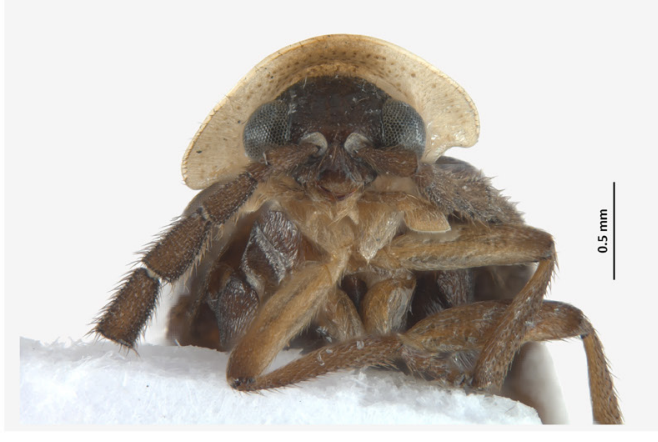

6

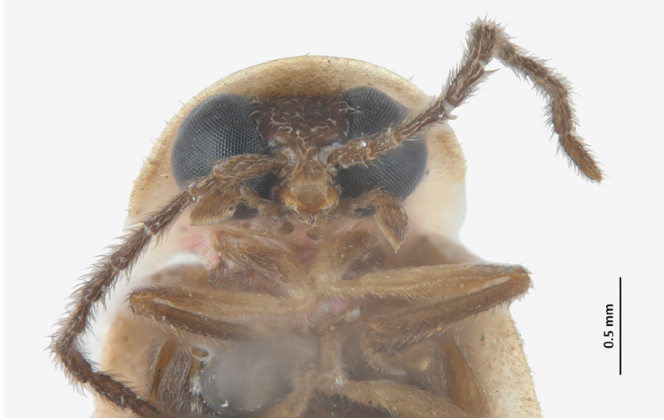

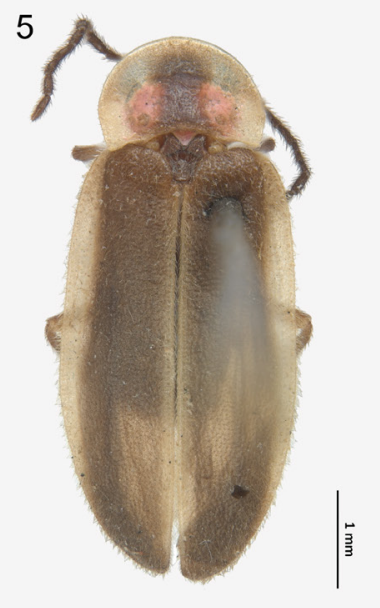

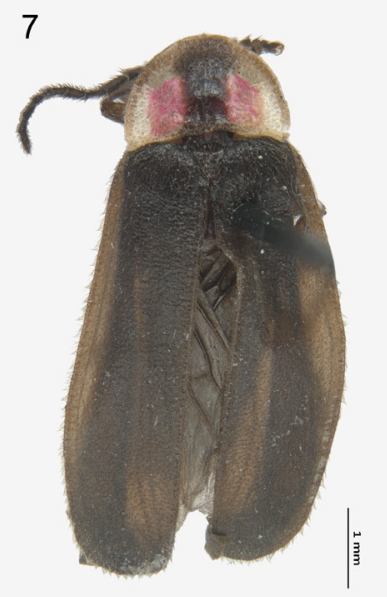

4

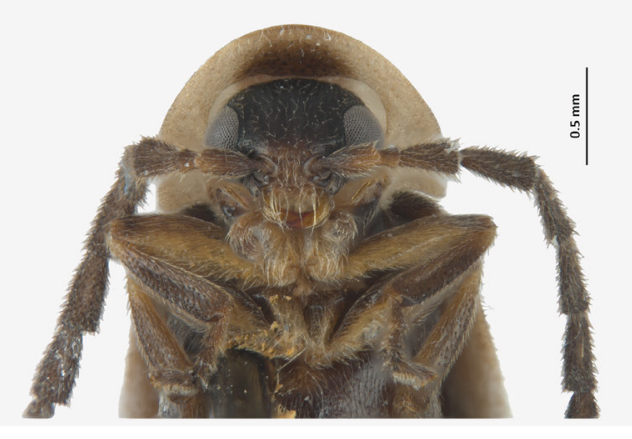

8

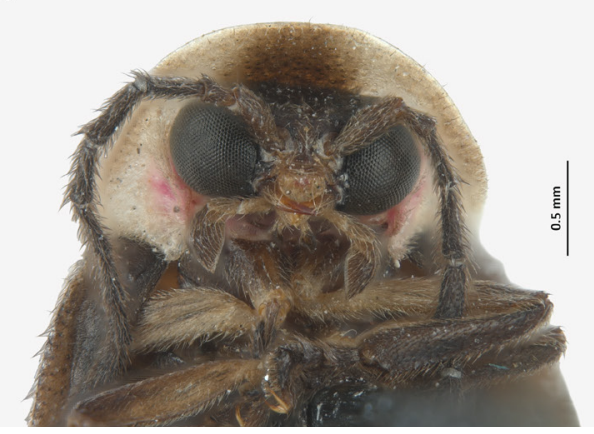

Figuras 1-8. Vista dorsal y frontal de:1-2 Aorphallus cibriani sp. nov.; 3-4 Ankonophallus consueloae sp. nov.; 5-6 Paraphotinus acremophallus sp. nov.; 7-8 $P$. aulophallus sp. nov. Figuras 9-20. Vista dorsal, ventral y lateral del edeago de: 9-11 Aorphallus cibriani sp. nov.; 12-14 Ankonophallus consueloae sp. nov.; 15-17 Paraphotinus acremophallus sp. nov.; 18-20 P. aulophallus sp. nov. y 21-23 Paraphotinus furcatus Zaragoza. 


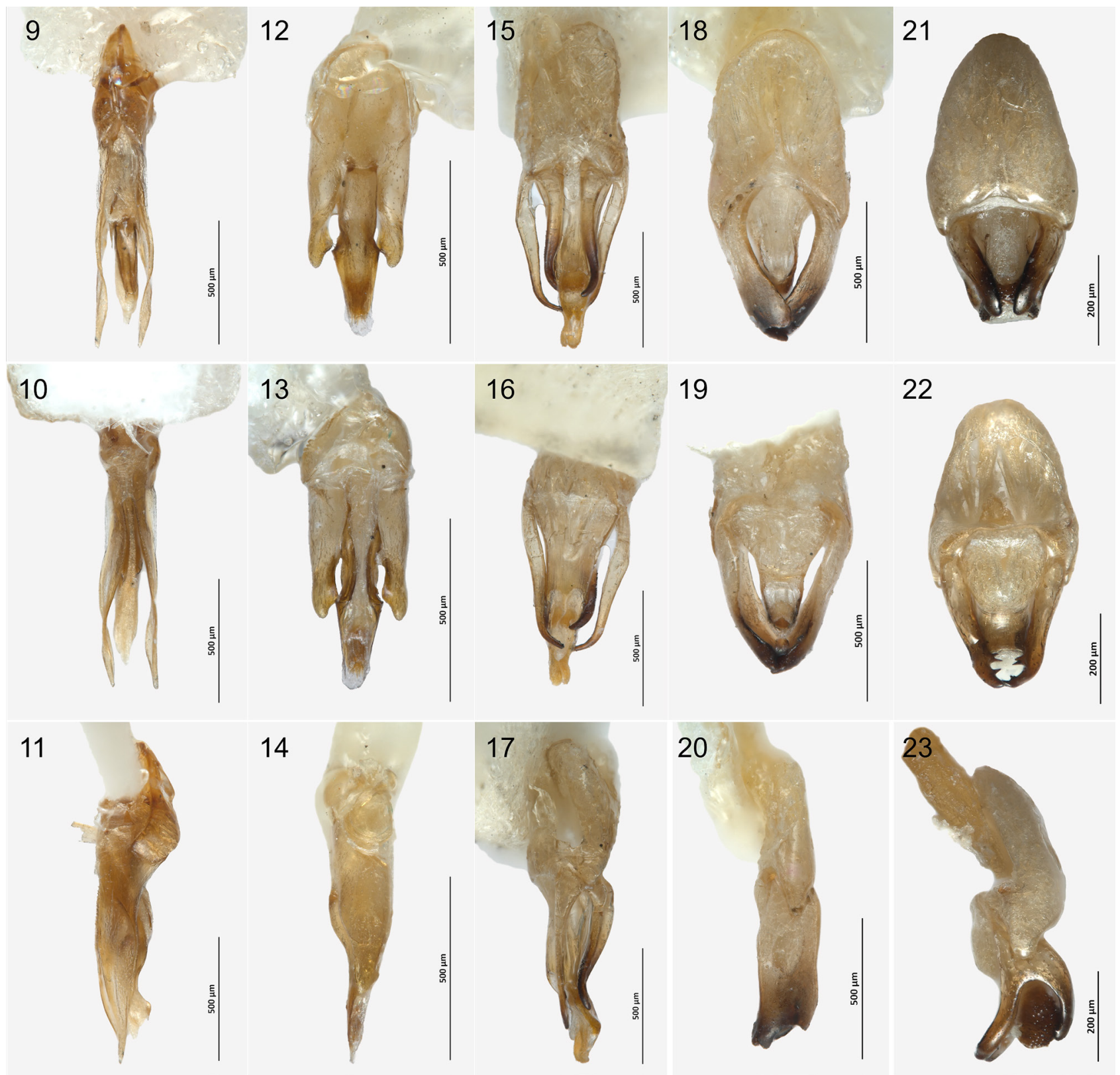

Figuras 9-20. Vista dorsal, ventral y lateral del edeago de: 9-11 Aorphallus cibriani sp. nov.; 12-14 Ankonophallus consueloae sp. nov.; 15-17 Paraphotinus acremophallus sp. nov.; 18-20 P. aulophallus sp. nov. y 21-23 Paraphotinus furcatus Zaragoza.

Apéndice 1. Especies del subgénero Paraphotinus Zaragoza, 1995

Paraphotinus acremophallus sp. nov.

Paraphotinus acuminatus Green, 1956

Paraphotinus anisodrilus Zaragoza, 2007

Paraphotinus aulophallus sp. nov.

Paraphotinus cookii Green, 1956

Paraphotinus curtatus Green, 1956

Paraphotinus floridanus Fall, 1927
Paraphotinus furcatus Zaragoza, 2000

Paraphotinus immaculatus Green, 1956

Paraphotinus juarezae Zaragoza, 1996

Paraphotinus marginellus LeConte, 1852

Paraphotinus sabulosus Green, 1956

Paraphotinus texanus Green, 1956

Paraphotinus tlapacoyaensis Zaragoza, 1996

Paraphotinus tuxtlaensis Zaragoza, 1995 